\title{
A Methodology for Speech Quality Assessment in Wireless Networks
}

\author{
Jinendra Dharmapala and Dileeka Dias
}

\begin{abstract}
Petformance factors are important in today's competitive telecommunications market where price differentials have been minimized. An important aspect of service performance is speech quality. This paper illustrates the theoretical foundation of Perceptual Evaluation of Speech Quality (PESQ), the most recent ITU-T standard for objective assessment of speech quality in telecommunication networks, and a methodology for its implementation in wireless networks.
\end{abstract}

Further, the development of the PESQ algorithm according to the standard is presented with the methodology of obtaining the PESQ scores which measure the perceived speech quality of the telecommunication network under test. Finally, the results obtained with the developed measurement setup are discussed. How the results can be used to analyse the impact of various factors on the speech quality of wireless networks is explained. The results were also used to examine the correlation between channel conditions (e.g. network load and signal strength) and the perceived speech quality.

\section{Introduction}

In the past few decades, wireless telephony networks have shown an exponential growth in subscribers, reach and complexity. At present, in the highly competitive telecommunication industry in which the price and technological differentials have been minimized, speech quality becomes a key market differentiator. A reliable prediction of quality can also be very useful in improving the network performance with new infrastructure planning and for optimizing the network parameters in order to meet the expected end-to end quality.

In the early stages of telecommunication networks, speech quality could be predicted from signal processing metrics such as frequency response, noise and delay.

However, the recent technological advances have introduced compressive coding techniques, level adjustment, echo cancellation and silence detection etc. which render conventional speech quality assessment techniques unsuitable. Therefore, there has been continuous growth in speech quality assessment technologies which are capable of accounting for numerous factors affecting the quality in modern networks such as filtering, variable delay, distortions due to variable channel conditions or poor received signal strength. These factors are more significant in wireless networks than in the wired networks.
A new generation of objective speech quality assessment techniques has been developed to take these complex factors into consideration when predicting the perceived quality of speech in telecommunication networks. The Perceptual Evaluation of Speech Quality (PESQ) is the latest ITU-T standard (ITU-T P.862) [1] for end-to-end speech quality assessment of narrow-band telephone networks and codecs. Such standards have become increasingly important in recent times due to the proliferation of packet-switched voice techniques and wireless networks, where speech quality degradation is not uncommon.

\section{Fundamentals of speech quality assessment tools}

Until recently the only way to measure users' perception of the quality of speech in telecommunication networks was to conduct a subjective test in which subjects hear recordings degraded by candidate networks, and vote on their opinion of the quality of each recording. The benchmark of quality is the opinion scale against which the subjects vote; an example, listening quality, is given in Table 1. Votes are averaged across subjects to give a mean opinion score (MOS). The MOS reflects the perceived performance of the whole network [2].

Eng. Prof. Ms. Dileeka Dias, B. Sc. (Eng.), M.S., PhD.

MIE (Sri Lanka) Department of Electronic \&

Telecommunication Engineering, Lniversity of Moratuwa.

Eng. Ms. Jinendra Dhannapala, B.Sc. Eng., AMIE (Sri Lonka) 
Table 1: Listening Quality Opinion Scale

\begin{tabular}{|l|c|}
\hline \multicolumn{1}{|c|}{ Quality of the speech } & Score \\
\hline Excellent & 5 \\
\hline Good & 4 \\
\hline Fair & 3 \\
\hline Poor & 2 \\
\hline Bad & 1 \\
\hline
\end{tabular}

However, subjective tests are expensive and slow, and cannot be used in certain applications such as in-service monitoring.

Therefore objective models, based on human perception, have been developed with the aim of predicting the results of subjective tests. Recent advances in perceptual modelling have led to the development of auditory perceptual models. These models can be used to generate predictions of perceived speech quality from a customer's perspective [11][12].

Several auditory perceptual models have been standardised by the ITU- T such as Perceptual Speech Quality Measure (PSQM), Perceptual Analysis/ Measurement System (PAMS) Measuring Normalizing Blocks (MNB) and Perceptual Evaluation of Speech Quality (PESQ) [3] [10].

All these models are based on the general structure in which both the input and output signals are converted into the psychoacoustic domain through a series of non linear mathematical processing which is similar to the internal representation of human audition as shown in Figure 1.
Perceptual Speech Quality Measure (PSQM) is one of the earliest perceptual models developed for measuring speech quality. The purpose was to objectively measure the quality of telephone narrow band $(300-3400 \mathrm{~Hz})$ speech signals transmitted though different codecs under different controlled conditions [13]. PSQM was also standardized as ITU-T Recommendation P 861. The output of PSQM measures the 'perceptual distance' between the auditory transformations of the input and output signals. The PSQM score was mapped to a MOS-score which showed low correlation with subjective results due to poor mapping and the timealignment functions. This was further developed to address severe distortion conditions and time clipping and was then known as PSQM 99.

The emphasis in the development of Perceptual Analysis/Measurement System (PAMS) has been end-to-end assessment of real networks. The previous perceptual models such as PSQM were mainly intended for the assessment of telephone-bandwidth codecs and proved unreliable in end-to-end measurement applications. Problems arise from the measurement interface, which may include significant analogue filtering, and from the complex behaviour of recent voice technologies - variable delay in Voice over IP (VoIP) being an important example. PAMS achieves this by the following elements of the model. Time alignment algorithm accounts for variable delay, particularly a problem with voice over IP networks. Level alignment and equalization deal with interface and analogue effects, essential if the model is to be used outside the digital domain. The modified structure for PAMS is shown in Figure 2.

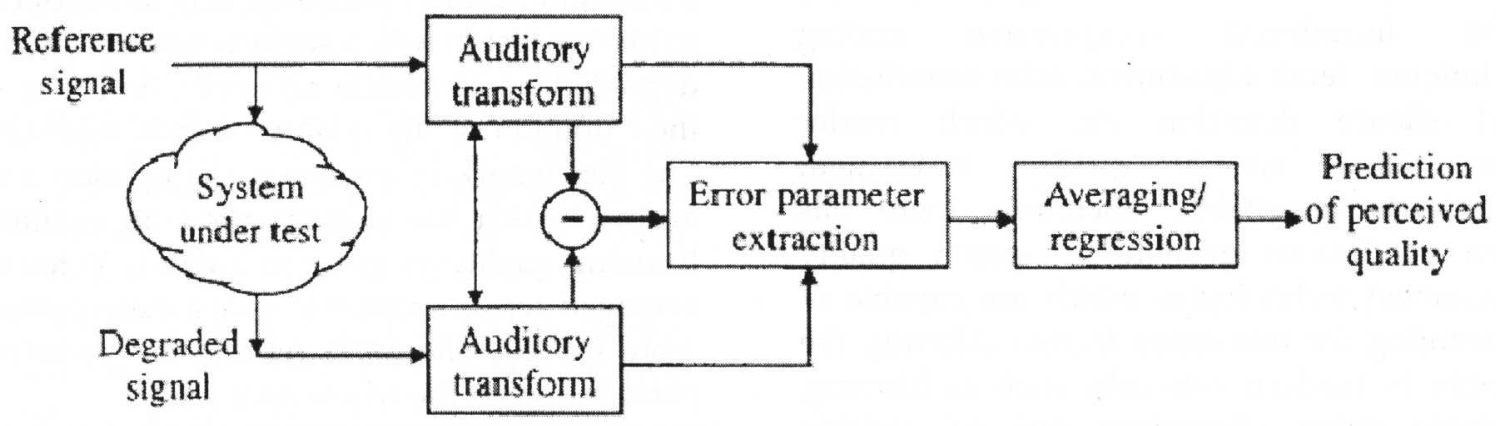

Figure 1: Basic Structure for Auditory Perceptual Models [3] 


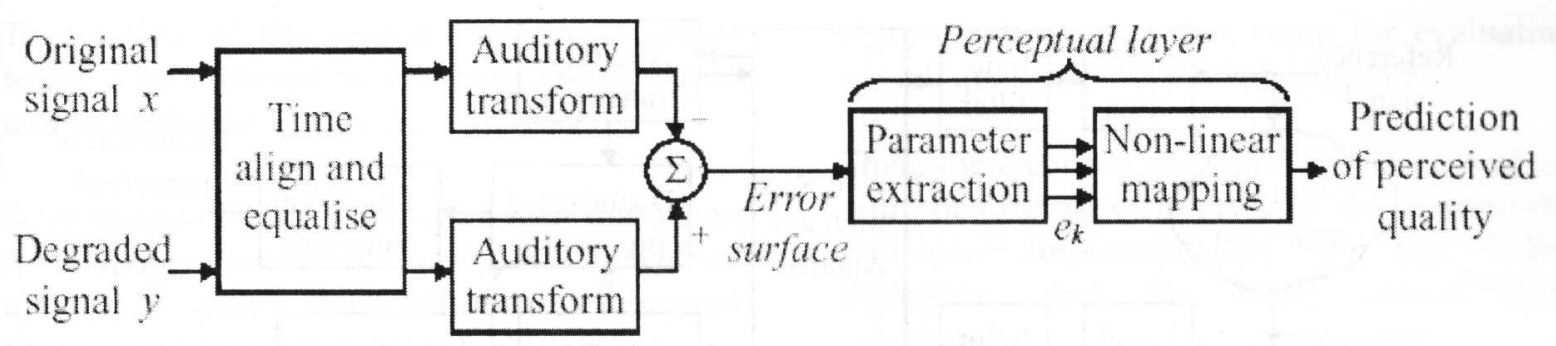

Figure 2: Structure of PAMS [4]

MNB (Measuring Normalizing Blocks) was proposed as an alternative method to PSQM and was accepted as an appendix to recommendation P.861. In this method the perceptual distance between the original and degraded signals were measured in a different way to that of PSQM. It was observed that listeners perceive spectral variations that span different time and frequency scales differently. MNB addressed this issue by analysing perceptual distance across multiple time and frequency scales. The MNB technique is capable of analysing the effect of the following factors on speech quality.

- Transmission channel errors

- Vocoders (Voice codecs which use frequency domain techniques)

- CELP and hybrid codecs with bit rates $<4$ kbps

Among the large number of speech quality assessment methods developed in the recent past, the Perceptual Evaluation of Speech quality (PESQ: ITU-T P 862), the latest ITU-T standard for speech quality assessment is capable of taking into account most of the complex aspects such as filtering, variable delay and short localized distortions.

The basic structure shown in Figure 1 has been modified in PESQ in order to address the following key effects which made the previous models unreliable [5].

- Variable delay

- Linear filtering,

- Gain variation

- Temporal clipping

These were caused by different network conditions such as packet-switched networks and were not properly taken into account in the previous objective models described above.
The first step in PESQ is the alignment of of both signals to a standard listening level. They are filtered (using a Fast Fourier Transform) with an input filter to model the telephone handset. The signals are aligned in time and are then processed through an auditory transform similar but enhanced to that of PSQM. Part of the transformation involves equalizing the signals for the frequency response of the system and for gain variation.

The difference between the transforms of the reference and degraded signals is known as the disturbance. This gives a measure of audible error. When this difference is positive, components such as noise have been added. When it is negative, components have been omitted from the original signal. This difference array is called the raw disturbance density. The process of small differences being inaudible in the presence of loud signals (masking) in each time-frequency cell is modelled using a two dimensional array called 'mask array' which is calculated using the minimum of the original and degraded loudness densities. The mask value is subtracted from the absolute loudness difference, and values less than zero are set to zero [5]. A deletion (a negative delay change) leaves a section which overlaps in the degraded signal. If the deletion is longer than a half a frame, the overlapping sections are discarded. PESQ computes two different error averages, one without and one with an asymmetry factor. (This asymmetry factor equals the ratio of the distorted and original pitch power densities raised to the power of 1.2[1]). These two distortion parameters, which are aggregated in frequency and time and mapped to a prediction of subjective MOS-like score between 1.0 and 4.5 , the normal range of MOS value given in Table 1. The modified structure of PESQ is shown in Figure 3[6]. 


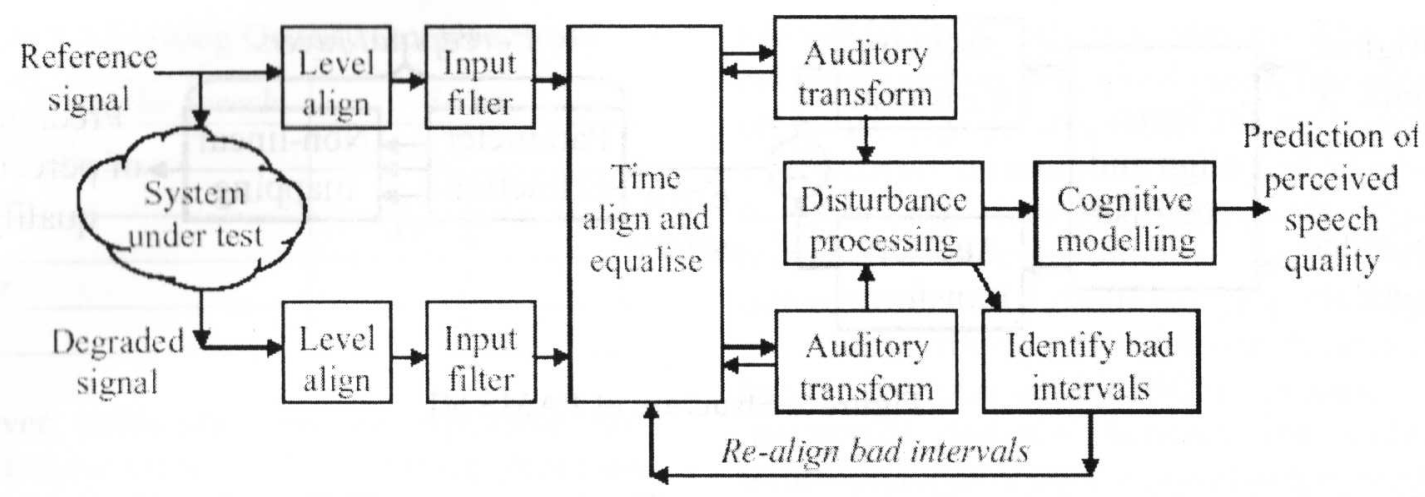

Figure 3: Structure of PESQ [6]

\section{Reference Implementation of PESQ}

As explained in the previous section, PESQ shows better correlation with the results of the subjective tests in predicting subjective quality in narrow-band telephone networks. After a detailed study on the algorithm to identify the key functional blocks of the PESQ algorithm, a software program was compiled using the files provided in ANCI-C reference implementation of PESQ which forms an integral part of the recommendation ITU=T P 862 [1].

It is important that test signals for use with PESQ are representative of the real speech signals carried by communications networks. Networks may treat speech and silence differently and coding algorithms are often highly optimised for speech and so may give meaningless results if they are tested with signals that do not contain the key temporal and spectral properties of speech. Therefore, in validating the implementation of PESQ algorithm, 40 speech file pairs were used from ITU standard speech file data base [1]. These files are in .wav format (16 bit linear PCM, Intel byte ordering, 44 - byte header) at $8 \mathrm{kHz}$ sampling rate. All files were as long as $8 \mathrm{~s}$ and contained speech as well as silence periods within each file. Also the speech files contained both male and female speech.

First, a general perception of quality of the original speech files was obtained by listening. The degraded files contained combination of various conditions affecting quality; variable delay, filtering, gain variations and temporal clipping.
Then the file pairs were given as the input to the software program one by one to obtain the PESQ scores, resembling the perceived quality. The PESQ scores obtained were compared with the values provided with the standard and it was confirmed that the Reference Implementation complies with the standard P. 862.

The software program was further verified with simulated error conditions typically found in wireless networks.

For this software modules from ITU-T Standard Tool Library 2000 were used in developing a program (Error Insertion Device) [7] which can introduce the error conditions to the original speech file.

Since the main focus of the study was predicting the speech quality in wireless channels, the Error Insertion Device (EID) was developed for simulating the errors encountered in wireless channels. For this, an error generating algorithm were required. The EID module was implemented with the following functionalities;

- Burst or random bit error generator.

- Random and bursty frame erasures.

Then, the effect on speech quality was studied by obtaining the PESQ scores with different error rates using the same set of speech files. When the same set of speech files were inserted with random bit errors and the burst frame errors (BFER) it was observed that burst frame errors have a grater impact on the perceived quality of speech than that of random bit errors. With the burst frame errors, the PESQ score dropped in the range of $0.5 \sim 1.5$. 
The quality of the speech files were further tested with different bit error rate (BER) values and Burst Frame Erasure Rate (BFER).

According to Figure 4 it can be seen that when the error patterns are bursty the perceived quality of speech drastically reduces beyond the acceptable speech quality level of 3.0.

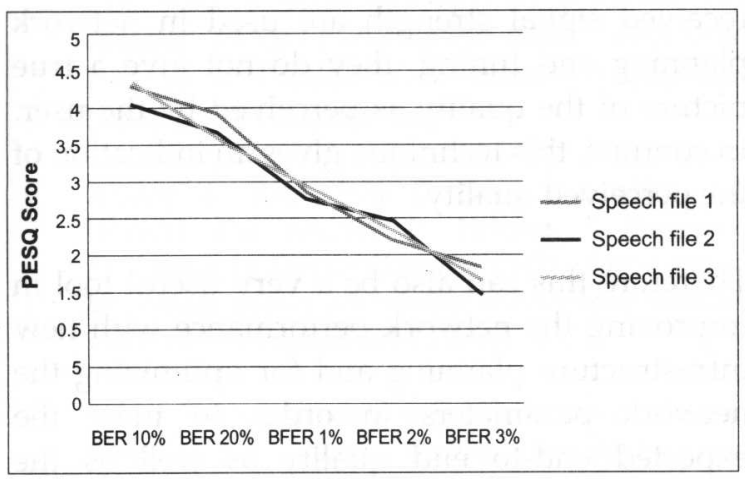

Figure 4: PESQ score Vs error rates

\section{Speech Quality assessment in a CDMA network}

A platform for evaluating speech quality in real wireless networks was developed based on the Reference Implementation of PESQ software discussed in the previous section.

In this experiment, the original speech files were transmitted from a stationary location and the degraded speech files were received and recorded at different locations and at one hour time intervals throughout the day. Two CDMA phones were also used at the transmit and receive ends to simulate listening through a handset with a frequency response that follows an IRS (Intermediate Reference System) receiver [8].
Figure 5 depicts the test setup for evaluating speech quality in the CDMA Network.

Then, the recorded degraded speech files along with the original were given as the input to the reference implementation of the PESQ algorithm, and the PESQ scores were calculated.

Nearly 200 degraded speech files were analysed in order to predict the effect of the following factors on the speech quality:

1. Network Load

2. Signal Strength/ Rx Power.

With the degraded (received over the wireless network) speech files recorded at different times of the day, speech quality values were calculated using the reference implementation of PESQ. Figure 6 shows the speech quality variations with the time.

According to Figure 6, it can be observed that during day time, the measured speech quality takes moderate values and it is improved at the time of less traffic in the network (off peak).

Therefore the drop in a quality of speech is experienced with the increasing load in the network. The poor PESQ scores resulted in some instances can be predicted to be due to 'cell breathing' phenomena in the CDMA networks at the border of the coverage area. This is due to the reduction in received power levels with the increased load in the network at the far ends of the cell area.

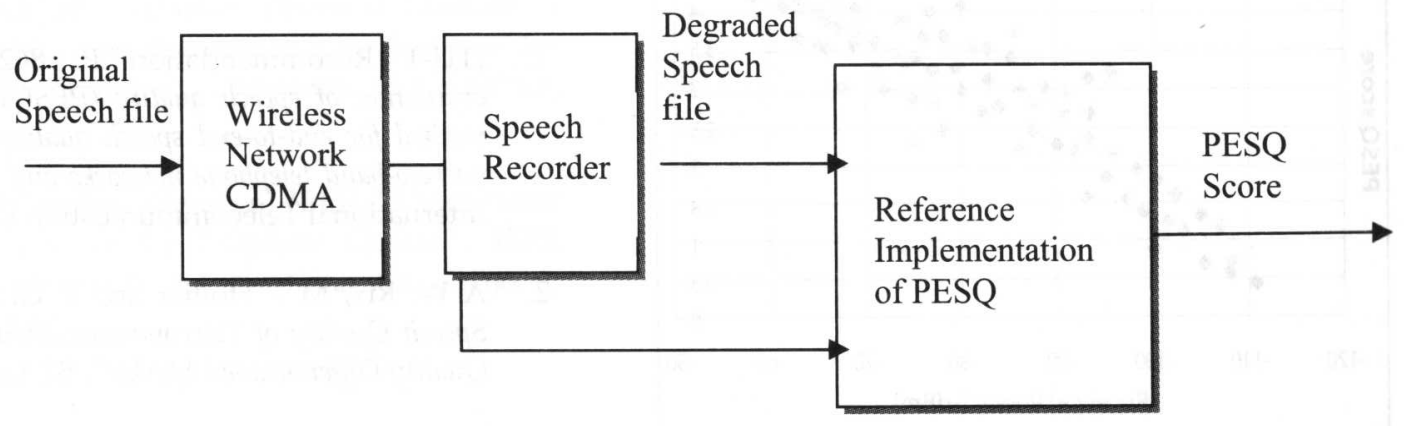

Figure 5: Block Diagram of the test setup for evaluating speech quality in a CDMA Network 


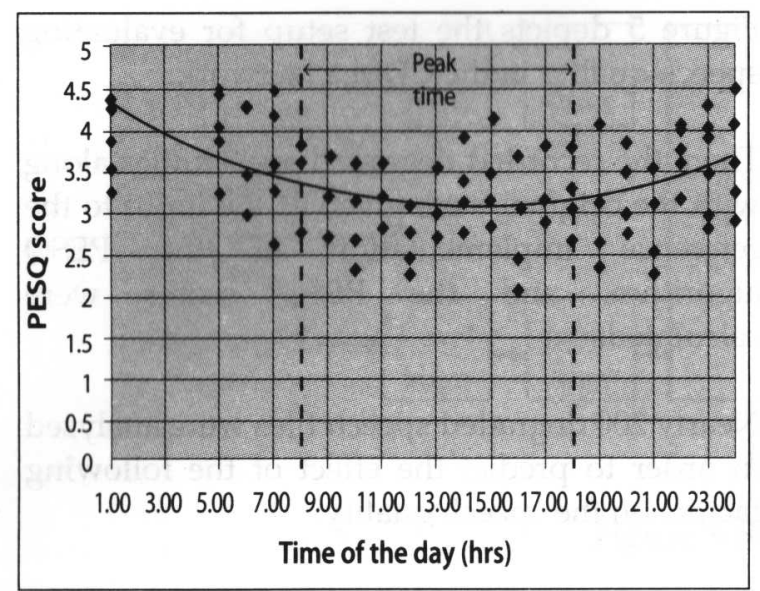

Figure 6: PESQ score Vs Time of the day

Speech quality variations in the CDMA network were further analysed with the received signal power at the CDMA phone at the end which the degraded file was recorded.

After obtaining the speech quality values for each degraded speech file using PESQ, Figure 7 shows the 'Speech quality' as a function of 'Received Signal Strength'.

In the Figure 7 it can be seen that though the threshold of the receiver is $-110 \mathrm{dBm}$, the perceived quality of speech at this level is very poor. Also, when the received signal level falls below - $95 \mathrm{dBm}$, the PESQ score drops below 2.5 , which can also be predicted to have around BER $>20 \%$ and BFER $>1 \%$ according to the analysis in the section 3 . This confirms that in the areas with poor radio coverage (radio signal strength being near to the receiver sensitivity threshold) the obtainable voice quality will be significantly degraded.

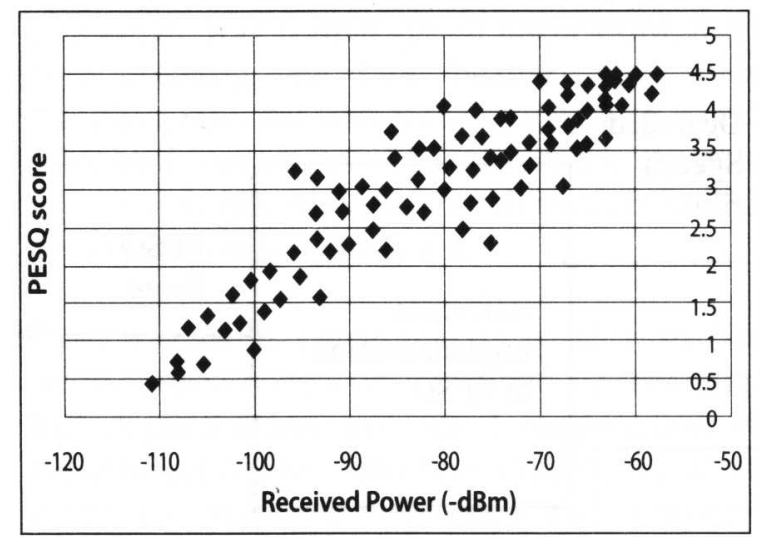

Figure 7: Speech Quality as a function of Received Signal Level

\section{Conclusion}

The methodology explained above can be used to obtain an indication of Quality of Service (QoS) in a wireless network based on the speech quality measurements.

Though objective measurements such as received signal strength are used in network planning and tuning, they do not give a true picture of the quality as perceived by the user. In contrast, this technique gives an indication of the perceived quality.

Therefore this can also be a very useful tool in improving the network performance with new infrastructure planning and for optimizing the network parameters in order to meet the expected end-to end quality as well as the quality of speech.

\section{Recommendations for Future Work}

The same methodology explained in this paper can be extended for speech quality assessment in different wireless networks and for assessing speech codecs.

This paper explains the analysis of only limited factors to see the impact of them on speech quality. Similarly the variations in speech quality can be analyzed with several other factors that might affect quality (for example, fading in wireless channels) [9]. Evaluating the correlation between the quality and error factors can be utilized in remedying quality issues and improving the network performance.

\section{References}

1. ITU-T Recommendation P. 862," Perceptual evaluation of speech quality (PESQ): An objective method for end-to-end speech quality assessment of narrow-band telephone networks and speech codecs" International Telecommunication Union, 2001.

2. A W. Rix, M P Hollier and P Gray "Predicting Speech Quality of Telecommunication Systems in a Quality Differentiated Market", BT Laboratories.

3. Antony W. Rix, Michael P. Hollier, Andries P. Hekstra, and John G. Beerends "Perceptual Evaluation of Speech Quality (PESQ), the new ITU standard for end-to-end speech quality assessment. 
Part I - Time alignment", Psytechnics Limited, BT Laboratories.

4. Antony Rix , Richard Reynolds and Mike Hollier "Robust Perceptual Assessment of End-toEnd Audio Quality", BT Laboratories, United Kingdom, Oct 1999.

5. Antony W. Rix, Michael P. Hollier, Andries P. Hekstra ,and John G. Beerends "Perceptual Evaluation of Speech Quality (PESQ), the new ITU standard for end-to-end speech quality assessment. Part II - Psychoacoustic model", Psytechnics Limited, BT Laboratories.

6. Antony W. Rix1, John G. Beerends2, Michael P. Hollierl and Andries P. Hekstra, "Perceptual Evaluation of Speech Quality(PESQ)-A new method for speech quality assessment of telephone networks and codecs", Psytechnics Limited,2002.

7. ITU-T Recommendation G 191," Software Tools for Speech and Audio Coding Standardizations" International Telecommunication Union, 2000.

8. A. Kajackas, A. Anskaitis "Investigation the Ability of Objective Measures of the Perceptual Speech Quality in Mobile Networks" Department of Telecommunications Engineering, Vilnius Gediminas Technical University, White paper,2002.

9. A. Kajackas, A. Anskaitis, D. Gursnys, L. Pavilanskas, "Estimation of QoS Dynamics in the Wireless Networks" Department of Telecommunications Engineering, Vilnius Gediminas Technical University, 2005.

10. Antony W. Rix and Michael P. Hollier,"The perceptual analysis measurement system for robust end-to-end speech quality assessment", BT Advanced Communications Research, June 2000.

11. P.Gray, M.P.Hollier and R.E.Massara, "Nonintrusive speech-quality assessment using vocal-tract models" white paper.

12. Holger Quast "Absolute Perceived Loudness of Speech", Machine Perception Lab, Institute for Neural Computation, University of California, San Diego.

13. Tomas G"ansler, Member, IEEE "Nonintrusive Measurements of the Telephone Channel", IEEE Transactions on communications vol. 47, no. 1, January 1999. 